\title{
Silica research in Glasgow
}

\author{
B W Barr ${ }^{1}$, G Cagnoli ${ }^{1,7}$, M M Casey ${ }^{1}$, D Clubley ${ }^{1}$, D R M Crooks ${ }^{1}$, \\ K Danzmann ${ }^{2,3}$, E J Elliffe ${ }^{1}$, S Goßler ${ }^{2}$, A Grant ${ }^{1}$, H Grote ${ }^{3}$, \\ A Heptonstall ${ }^{1}$, J Hough ${ }^{1}$, O Jennrich ${ }^{1}, \mathbf{H}$ Lück $^{2,3}$, S A McIntosh ${ }^{1}$, \\ G P Newton ${ }^{1}$, D A Palmer ${ }^{1}$, M V Plissi ${ }^{1,4}$, D I Robertson ${ }^{1}$, \\ N A Robertson ${ }^{1}$, S Rowan ${ }^{1,6}$, K D Skeldon ${ }^{1}$, P Sneddon ${ }^{1}$, K A Strain ${ }^{1}$, \\ C I Torrie ${ }^{1}$, H Ward ${ }^{1}$, P A Willems ${ }^{1,5}$, B Willke ${ }^{2,3}$ and W Winkler ${ }^{3}$ \\ ${ }^{1}$ Physics \& Astronomy, University of Glasgow, Glasgow G12 8QQ, UK \\ ${ }^{2}$ Institut für Atom- und Molekülphysik, Universität Hannover, Callinstr. 38, 30167 Hannover, \\ Germany \\ ${ }^{3}$ Max-Planck-Institut für Quantenoptik, Hans-Kopfermann-Str. 1, 85748 Garching, Germany \\ and Außenstelle Hannover, Callinstr. 38, 30167 Hannover, Germany \\ ${ }^{4}$ Max-Planck-Institut für Gravitationsphysik, Albert-Einstein-Institut, Am Mühlenberg 1, 14476 \\ Golm, Germany \\ ${ }^{5}$ LIGO Project, California Institute of Technology, Pasadena, CA, USA \\ ${ }^{6}$ Stanford University Gravitational Waves Group, Ginzton Laboratory, Stanford University, \\ Stanford CA, 94305-4085, USA \\ E-mail: g.cagnoli@physics.gla.ac.uk
}

Received 2 October 2001, in final form 24 October 2001

Published 14 March 2002

Online at stacks.iop.org/CQG/19/1655

\begin{abstract}
The Glasgow group is involved in the construction of the GEO600 interferometer as well as in $R \& D$ activity on technology for advanced gravitational wave detectors. GEO600 will be the first GW detector using quasimonolithic silica suspensions in order to decrease thermal noise significantly with respect to steel wire suspensions. The results concerning GEO600 suspension mounting and performance will be shown in the first section. Section 2 is devoted to the present results from the direct measurement of thermal noise in mirrors mounted in the $10 \mathrm{~m}$ interferometer in Glasgow which has a sensitivity limit of $4 \times 10^{-19} \mathrm{~m} \mathrm{~Hz}^{-1 / 2}$ above $1 \mathrm{kHz}$. Section 3 presents results on the measurements of coating losses. R\&D activity has been carried out to understand better how thermal noise in the suspensions affects the detector sensitivity, and in section 4 a discussion on the non-linear thermoelastic effect is presented.
\end{abstract}

\section{Silica suspensions in GEO600}

The interferometer GEO600 is designed to detect gravitational waves above $50 \mathrm{~Hz}$. At such frequencies the internal thermal noise in the mirrors is expected to give the main limitation to the

7 Author to whom correspondence should be addressed. 
detector sensitivity. The other two relevant mechanical noises, seismic noise and suspension thermal noise, do not give a significant contribution due to the suspension isolation system and the relatively low frequencies and low mechanical losses of the suspension resonances. Creep in suspension fibres may introduce a significant continuous noise or generate spurious bursts in the detector. Measurements have been made on steel wires [1] for creep rate and events detection showing that the contribution of creep for steel suspension is not relevant. From the same authors a measurement on creep rate for a loaded silica fibre has been reported in [2]. Due to the low rate measured, continuous noise coming from creep should be well below the thermal noise level. A group at the Moscow State University is working on the direct measurement of creep events in silica fibres to tackle the problem of spurious bursts [3].

Energy loss mechanisms are sources of thermal noise. Due to contact friction, mirror suspension systems realized with steel wires give a major contribution to the energy loss in the internal dynamics of test masses. In order to reduce this energy loss and then reduce the thermal noise in the detection bandwidth, GEO600 uses a special bonding technique (silicate bonding) to attach mirrors to the suspension systems. In this technique small silica prisms are chemically bonded on flat surfaces machined on the barrel of each mirror (figure 1). Silica fibres are then welded onto these silica prisms realizing what is called a quasi-monolithic suspension. In this system all internal frictional mechanisms are avoided and the high quality factor of silica is then preserved.

Fibres have been pulled using a computer-driven machine. Silica rods (Suprasil 3) of $8 \mathrm{~cm}$ length, $5 \mathrm{~mm}$ diameter, are melted in a middle region using an oxy-hydrogen flame coming out from five nozzles. Then they are pulled for approximately $2 \mathrm{~cm}$ in order to create a section having a diameter of about $2 \mathrm{~mm}$. Finally, once this thin section is melted again the fibre is pulled to the length of $285 \mathrm{~mm}$. The first pulling makes the fibre neck sufficiently thin to allow easy cutting later on (figure 2). Several fibres were produced in the AEI Institute in Hannover and then transported to the GEO600 site in a special box.

The pulling machine used was electronically controlled in order to produce a set of fibres as homogeneous as possible in terms of diameter and length. Differences of the order of a few $\mathrm{mm}$ in length are recovered through the cutting process. So, the only relevant fibre parameter is the longitudinal spring constant which has been measured through the bouncing mode resonant frequency. In this measurement each fibre was suspended with a load of $1.4 \mathrm{~kg}$ which is exactly the nominal load expected in GEO600. The fibres used had resonant frequencies between 16 and $16.5 \mathrm{~Hz}$.

Due to the high quality silica used and high dilution factor, the violin modes' $Q$ s are foreseen to be of the order of $10^{8}$. This corresponds to a characteristic decay time of about 2 days for the first mode $(500 \mathrm{~Hz})$. Unexpected excitations of violin modes could compromise the locking performances for too long a time. In order to reduce this time all the fibres used in this first suspension were coated with a small amount of Teflon on two $5 \mathrm{~mm}$ long sections: one in the middle and the other at a third of the total fibre length. In this way a nonhomogeneous loss was created. The particular position of the coated sections was chosen to damp particularly the first modes while affecting the dynamics at frequencies below a hundred Hertz in a negligible way. Teflon was applied through the deposition of drops of a solution of Teflon AP in a solvent called Flourinert.

Fibre strength was tested at $5 \mathrm{~kg}$ after coating. After this, cutting was initiated by scratching the necks with a diamond file. The correct position of the scratches was fixed using a jig where the fibres were held. Length reproducibility is of the order of $0.5 \mathrm{~mm}$.

Each fibre was welded to the tip of a prism using the small neck left from the cutting. Again an oxy-hydrogen flame was used to melt the silica. Several types of hypodermic needles were used as nozzles. The upper neck was welded at first, holding the fibre with steel tweezers 


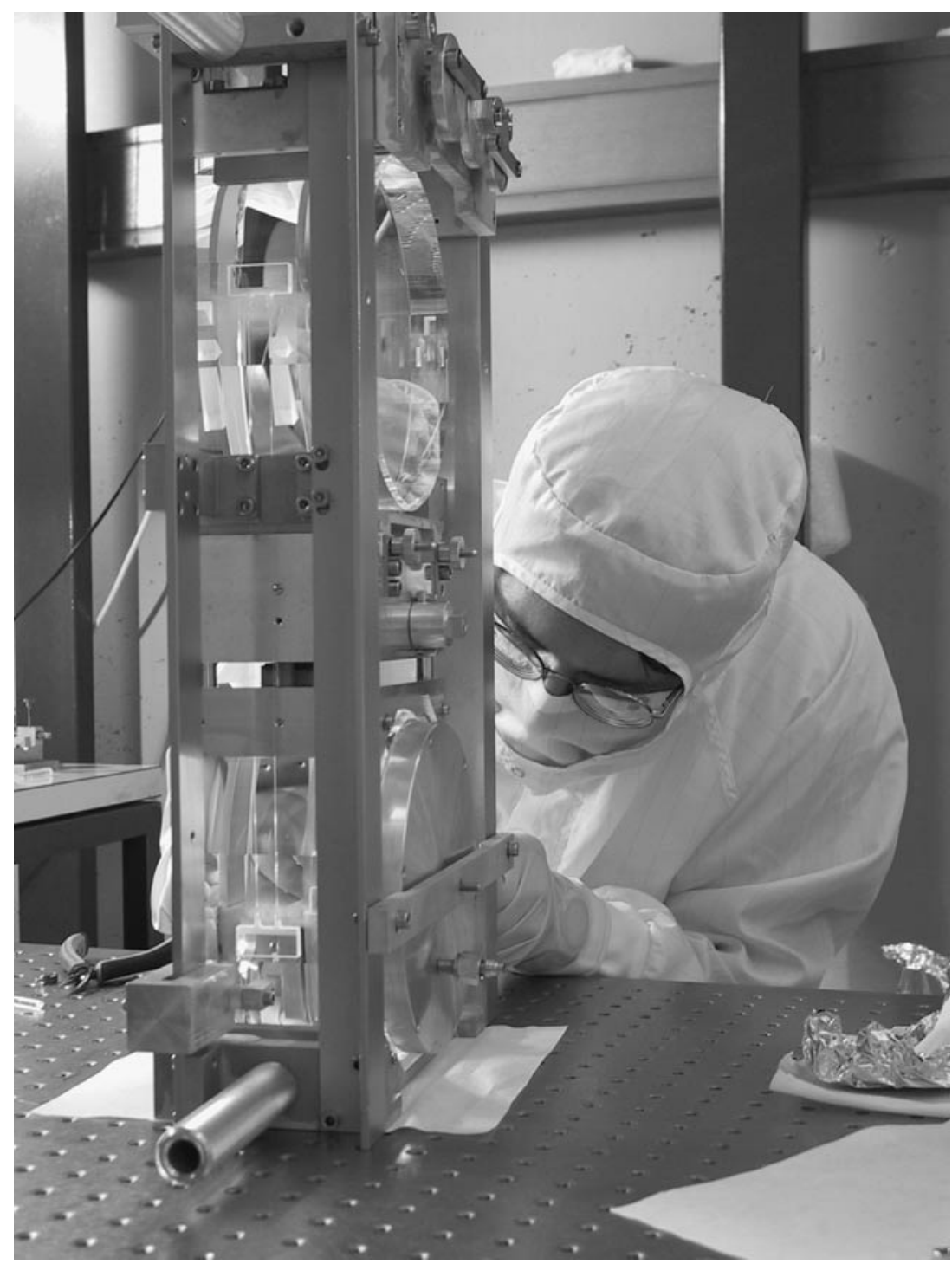

Figure 1. Intermediate mass on top and mirror at the bottom clamped in a steel frame. On the first plane the ears are bonded on both the masses and the two fibres welded.

as close to the neck as possible. Any resulting scratches were cured by melting the silica where the neck was held by the tweezers. Discrepancies between the fibre length and the distance between prism tips were fixed using melted silica from thin rods or by adjusting the vertical position of one neck using tweezers again.

\section{Direct measurement of thermal noise}

In the past year the Glasgow $10 \mathrm{~m}$ prototype interferometer with Fabry-Perot cavities in the arms has been upgraded using a high power IR laser $(800 \mathrm{~mW})$. As a result of this work, the sensitivity limit was pushed down below $10^{-18} \mathrm{~m} \mathrm{~Hz}^{-1 / 2}$ above $600 \mathrm{~Hz}$, as shown in spectrum 


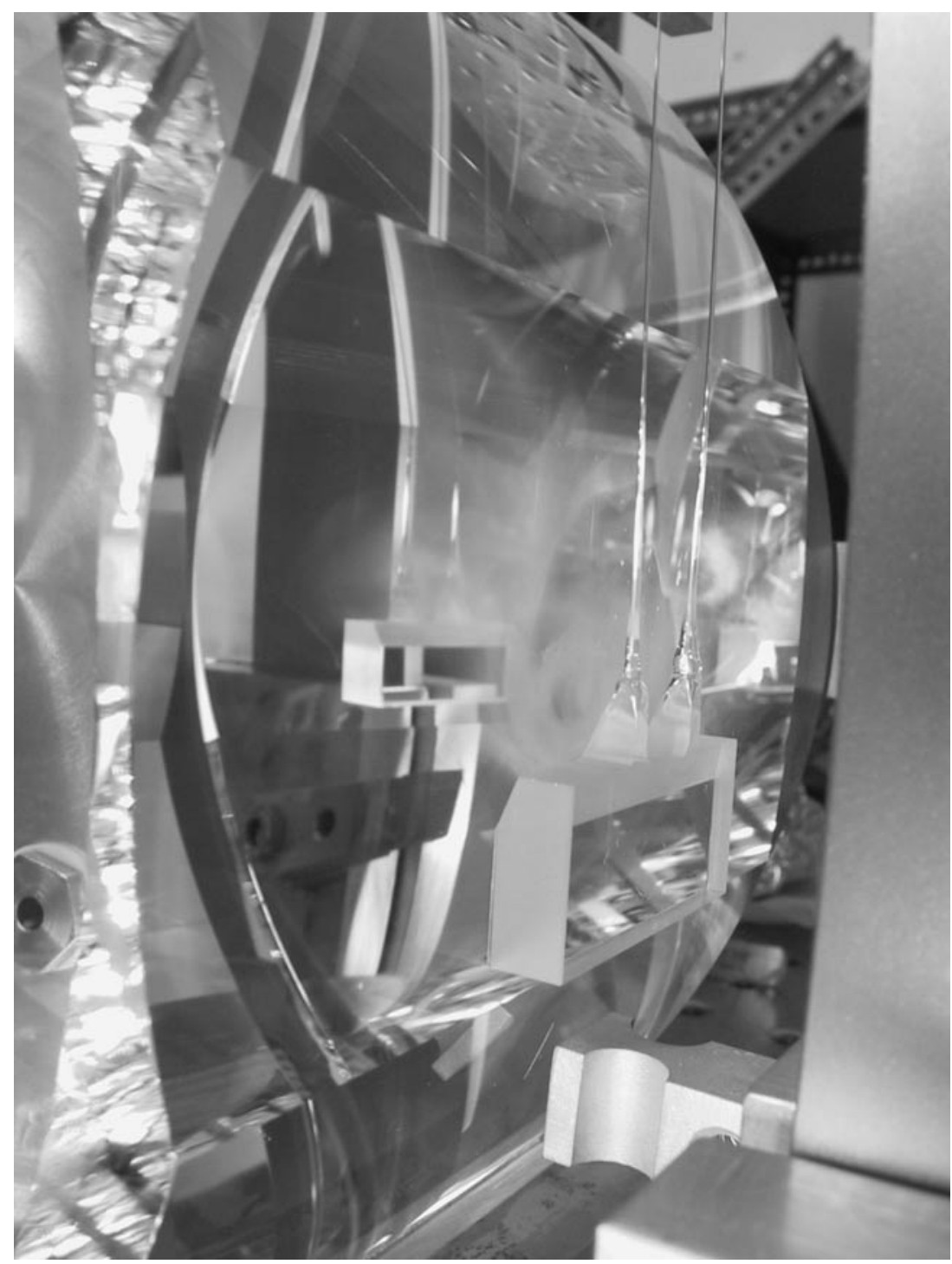

Figure 2. Detail of two fibres welded on the tips of a mirror ear.

(a) in figure 3. Several noise sources contributed to this limit and it was not possible to estimate them separately. In particular it was not clear how much displacement noise came from the thermal noise associated with the internal modes of the suspended optics.

In order to measure the internal thermal noise, it was decided to increase the internal damping of one of the mirrors using three strips of vacuum grease placed along the barrel, and then spectrum (b) in figure 3 was recorded. The time lag between the recording of spectrum (a) and spectrum (b) was kept as short as possible compatible with the re-making of vacuum in the tank. Clearly, an increase of the noise floor was seen suggesting that it may have come from the thermal noise of the greased mass.

$Q$ measurements of the greased mass were taken in another facility. In this facility the mass was suspended by a single steel wire. After the modes had been excited by an 


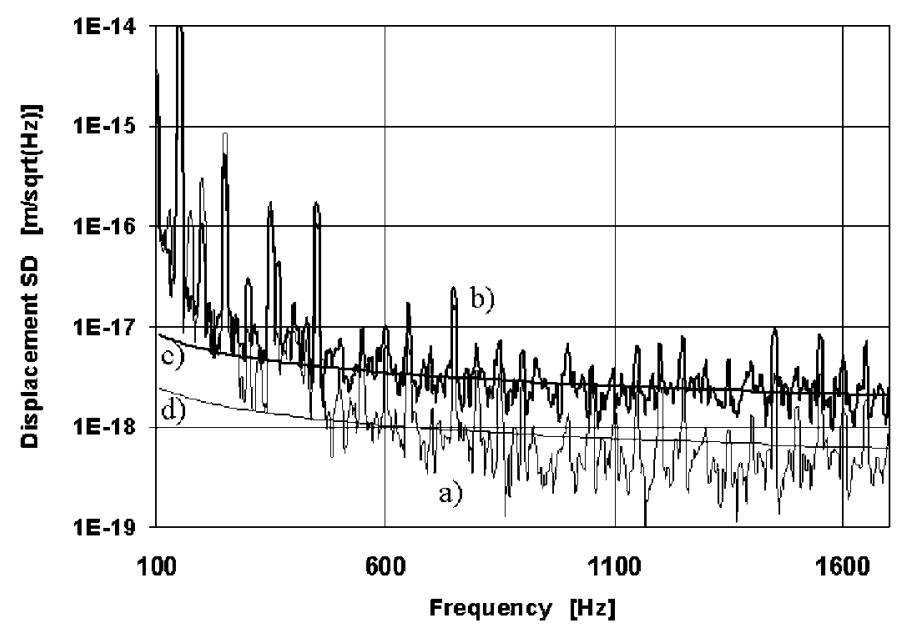

Figure 3. Displacement spectral densities. Curves (a) and (b) are recorded from the interferometer output before and after putting grease on one of the masses, respectively. Curves (c) and (d) are estimations using a $Q$ of 1800 for the greased mass and $1.2 \times 10^{5}$ for the others.

electrostatic actuator, the displacement of the front face was detected using a Michelson interferometer. More details about this facility can be found in [4]. Due to the short decay time of all the detected modes, $Q$ measurements were first taken by measuring the transfer function at frequencies close to the resonances and later the peak widths were extracted. Later, a recording oscilloscope was used to sample the ring down of each detected mode and then the decay time was derived. The transfer function measurement could be affected by the presence of split modes so close to each other as to not be measured as separate modes. The two sets of measurements gave consistent results. After removing the grease, $Q$ measurements were taken again.

Based on a $Q$ average value of 1800 for the greased mass and $1.2 \times 10^{5}$ for the other clean masses, the internal thermal noise was estimated using a semi-infinite homogeneousloss model as in [5]. The results of this calculation are shown in figure 3. The agreement is good but the theoretical model used is not correct in principle because the grease introduces a non-homogeneous loss. As showed by Yamamoto [6] thermal noise predictions based on a homogeneous loss model can differ substantially from those based on an inhomogeneous one. On the other hand, grease is a material having very different mechanical constants from those of silica, and the layer is so thick (about a few millimetres) that it may well be that the inhomogeneous model cannot be applied in a straightforward manner. Currently, research is being pursued in this area.

\section{Coating losses}

In a gravitational wave interferometric detector such as GEO600 the silica masses are coated in order to obtain the desired reflectivity. Different groups in the scientific community have started to measure or estimate the effect of this coating on the internal damping of the suspended masses [8,9]. A multilayer structure and the kind of materials used give sufficient reasons for a possible high loss characteristic of the coating. At the IGR in Glasgow we measured the loss factor of the seven lowest modes (figure 4) of a Corning 7980 coated mass (127 mm diameter, $100 \mathrm{~mm}$ length). The substrate loss is dominated by the structural damping that 


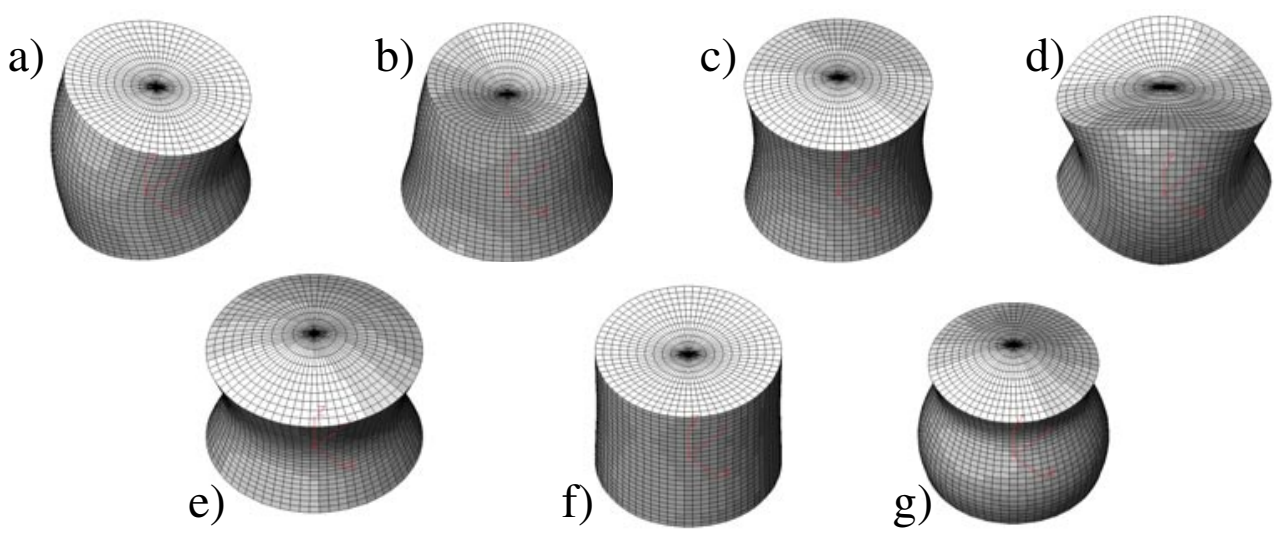

Figure 4. Shapes of the detected modes. (a) Bending, (b) first asymmetric, (c) fundamental, (d) clover, (e) symmetric, (f) expansion, (g) second asymmetric.

Table 1. Summary table showing results obtained for the Corning 7980 mass. Loss factor values are averages of measures taken with different suspensions. $C_{f f}$ is the front-face-to-bulk energy ratio. $C_{b}$ is the barrel-to-bulk energy ratio. Both are per $\mu \mathrm{m}$ coating thickness.

\begin{tabular}{lllll}
\hline Mode & $\begin{array}{l}\text { Frequency } \\
(\mathrm{Hz})\end{array}$ & $\begin{array}{l}\text { Loss factor } \\
\left(\times 10^{7}\right)\end{array}$ & $\begin{array}{l}C_{f f} \\
(\text { front face })\end{array}$ & $\begin{array}{l}C_{b} \\
(\text { barrel })\end{array}$ \\
\hline Bending & 22110 & $1.37 \pm 0.04$ & $1.67 \times 10^{-5}$ & $2.00 \times 10^{-4}$ \\
1st asymmetric & 22910 & $1.17 \pm 0.12$ & $1.23 \times 10^{-4}$ & $3.61 \times 10^{-5}$ \\
Fundamental & 25380 & $0.65 \pm 0.14$ & $1.39 \times 10^{-7}$ & $2.71 \times 10^{-5}$ \\
Clover & 25180 & $1.62 \pm 0.15$ & $3.56 \times 10^{-5}$ & $2.21 \times 10^{-4}$ \\
Symmetric & 28390 & $3.13 \pm 0.33$ & $1.05 \times 10^{-4}$ & $2.12 \times 10^{-4}$ \\
Expansion & 31710 & $1.09 \pm 0.19$ & $1.85 \times 10^{-5}$ & $8.13 \times 10^{-5}$ \\
2nd asymmetric & 36050 & $0.86 \pm 0.15$ & $2.94 \times 10^{-6}$ & $9.22 \times 10^{-5}$ \\
\hline
\end{tabular}

is independent of frequency. So, the differences between the loss factor of different modes have to be considered as a result of the coating effect. The loss properties of the coating are assumed to affect the total loss factor $\phi_{t}$ shown by a mode through the following relation:

$$
\phi_{t}=\phi_{0}+C_{f f} \cdot \phi_{c f}+C_{b} \cdot \phi_{c b}
$$

where $\phi_{0}$ is the substrate or bulk loss angle and $\phi_{c f}$ and $\phi_{c b}$ are the coating loss factors associated with the front face and with the barrel. In fact, during the coating process, some coating material spilled off and contaminated the barrel in an unknown way. In order to take into account this uncertainty, two different loss factors are assumed for the coating, one for the front face and one for the barrel. $C_{f f}$ and $C_{b}$ are the scaling factors and they are defined as front-face-to-bulk and barrel-to-bulk energy ratios, respectively. A proof of equation (1) for a general case is given in [10]. Since the energy ratios scale linearly with the coating thickness, at least for thin coatings, both the $C$ parameters are calculated per $\mu$ m coating thickness; values for each mode are listed in table 1. Finite element analysis was used for the mode shapes and a C-code was developed to calculate the energies.

When all the data were used in a three-parameter linear fitting, to solve equation (1), the resulting regression parameter $R$ was rather low. The best fitting was obtained by rejecting 


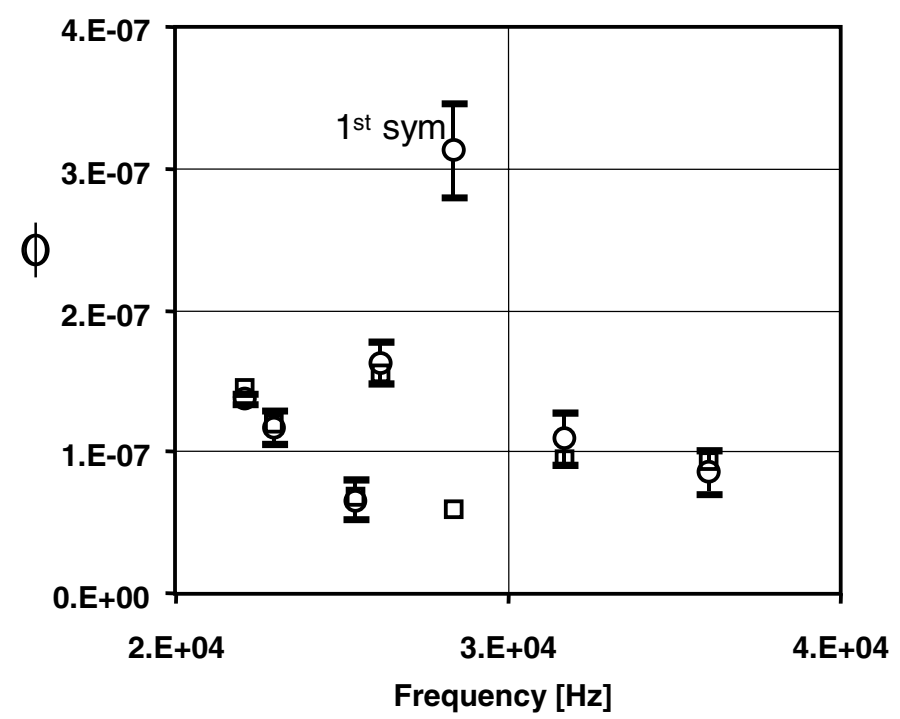

Figure 5. This plot shows the comparison between the measured loss factor for each mode (circles) and the estimated loss factor (squares) as they come from a three-parameter linear fitting. The symmetric mode data were removed from the fitting process because they show a significantly higher loss than the estimated one.

the data for the symmetric mode and the result is shown in figure 5. The agreement between expected values and the experimental points is within the experimental errors. The large discrepancy obtained for the symmetric mode may come from a suspension loss particularly effective for this mode.

Using the loss factors per unit thickness coming from the fitting function and knowing that the effective coating thickness was $6.3 \mu \mathrm{m}$, the following values for the loss factors are obtained:

$$
\begin{aligned}
& \phi_{0}=(5.6 \pm 0.9) \times 10^{-8} \\
& \phi_{c f}=(6.3 \pm 1.6) \times 10^{-5} \\
& \phi_{c b}=(6.3 \pm 0.9) \times 10^{-5}
\end{aligned}
$$

\section{Non-linear thermoelastic effect}

Thermal fluctuations inside a material produce strain fluctuations due to thermal expansion effects (coefficient $\alpha$ ). This mechanism is responsible for shape fluctuations of mirrors [5] and of suspension fibres that are seen by the interferometer as displacement noise. Thermal conduction makes this mechanism dissipative and it represents a fundamental process known as thermoelastic damping, studied first by Zener [7].

The mechanical loss angle determined by thermoelastic damping has the following general expression:

$$
\phi_{Z}=\alpha^{2} \frac{E T}{C_{V}} f(\omega)
$$

where $\alpha$ is the linear thermal expansion coefficient, $E$ Young's modulus, $T$ the temperature, $C_{V}$ the heat capacity per unit volume and finally $f(\omega)$ the frequency-dependent part that is 
defined by the dimensions of the sample and by the heat conductivity $K$ and capacity $C_{V}$ of the material used. The coefficient in front of $f(\omega)$ in the previous equation gives the strength of the damping. For silica this coefficient is about $3.6 \times 10^{-6}$, which is high compared to the structural loss factor which can be as low as $1 \times 10^{-7}$ in thin fibres. This comparison becomes more relevant if one considers that for silica fibres the thermoelastic pick has its maximum in the range of tens of $\mathrm{Hz}$ where the effects of the pendulum thermal noise are still relevant in comparison to those coming from the internal thermal noise of mirrors.

There is another mechanism to convert temperature fluctuations to strain $(\epsilon)$ fluctuations. In this mechanism the stress-strain relations have an explicit temperature dependence through their elastic coefficients. Young's modulus variations contribute to the strain field $(\sigma)$ as a second-order effect except when a large static stress is present in the material. This is the case for the suspension wires or fibres that are stressed by the weight of a test mass. For longitudinal deformations the stress-strain relation reads $\epsilon=\sigma / E$. If a variation $\delta E \ll E$ of Young's modulus is considered, then $\delta \epsilon \simeq-\epsilon(\delta E / E)$. In the case of a large static strain, from the previous relation, Young's modulus variation produces a first-order effect on the dynamical strain. Moreover the sign of $\delta \epsilon$ with respect to $\delta E$ depends on the static strain applied.

For a stressed body, then, one can talk about an effective thermal expansion coefficient $\alpha_{e}=\alpha-\beta\left(\sigma_{0} / E\right)$, where $\beta=(\mathrm{d} E / \mathrm{d} T) / E$ is the linear thermoelastic coefficient. It is possible to demonstrate [11] that the loss factor becomes

$$
\phi_{N L T}=\left(\alpha-\beta \frac{\sigma_{0}}{E}\right)^{2} \frac{E T}{C_{V}} f(\omega) .
$$

For silica $\beta=2 \times 10^{-4} 1 / \mathrm{K}, \alpha=5.5 \times 10^{-7} 1 / \mathrm{K}, E=72 \mathrm{GPa}$. Assuming these constants, the thermal expansion is completely compensated by Young's modulus variation with a static stress of only $\sigma_{0}=200 \mathrm{MPa}$. In this condition the overall thermoelastic effect is nullified.

\section{References}

[1] Cagnoli G et al 1997 Phys. Lett. A 23721

[2] Gammaitoni L et al 2000 Proc. 3rd Edoardo Amaldi Conf. on Gravitational Waves (Caltech) ed S Meshkov (New York: AIP) p 162

[3] Bilenko I A 2002 Proc. of the 4th Edoardo Amaldi Conf. on Gravitational Waves (Perth, Western Australia, 8-13 July 2001) Class. Quantum Grav. 192035

[4] Rowan S et al 2000 Phys. Lett. A 2655

[5] Liu Y T and Thorne K S 2000 Phys. Rev. D 62122002

[6] Yamamoto J K 2001 PhD Thesis webpage http://t-munu.phys.s.u-tokyo.ac.jp/tsubono_group_e.html\#theses

[7] Nowick A S and Berry B S 1972 Anelastic Relaxation in Crystalline Solids (New York: Academic)

[8] Crooks D R et al 2002 Class. Quantum Grav. 19883 (Crooks D R M et al 2001 Preprint gr-qc/0109074)

[9] Harry G M et al 2002 Class. Quantum Grav. 19897 (Harry G M et al 2001 Preprint gr-qc/0109073)

[10] Cagnoli G et al 2000 Phys. Lett. A 27239

[11] Cagnoli G and Willems P A Phys. Rev. D at press 